\title{
Towards cost-effective soil microbial fuel cell designs
}

\author{
Jakub Dziegielowski ${ }^{1,2}$ and Mirella Di Lorenzo ${ }^{1,2,}$ * \\ ${ }^{1}$ Department of Chemical Engineering, University of Bath, BA2 7AY, Bath, United Kingdom \\ ${ }^{2}$ Centre for Biosensors, Bioelectronics and Biodevices, University of Bath, BA2 7AY, Bath, United Kingdom
}

\begin{abstract}
Soil microbial fuel cell (SMFC) is a carbon-neutral energy harvesting technology that exploits the use of electroactive bacteria naturally present in soil to directly generate electricity from organic compounds. Given the simplicity of the system design, SMFCs have great potential to be used for decentralised solutions, especially in areas where access to conventional energy sources is limited. Yet, the high cost to power ratio severely limits the translation of this technology into the market. With the aim of reducing the capital cost, in this study we explore the effect of decreasing the amounts of current collector (CC) on the performance. The results demonstrate that increasing the amount of current collector per surface area of the electrode is not a feasible way of enhancing power densities, as to increase the performance by $20 \%$ and $35 \%$, the amount of current collector would have to be increased by $150 \%$ and $300 \%$, respectively. This highlights the importance of economic evaluations when optimising the design of a SMFC.
\end{abstract}

\section{Introduction}

Biomass energy has been fuelling the world for thousands of years, far long before the industrial revolution [1]. Since then, it has, however, been replaced by fossil fuels, an unsustainable resource, responsible for a lot of the damage to our climate. there is, therefore, the urgent need for alternative sources that can meet our energy demands with clean, safe and renewable solutions.

Microbial fuel cell (MFC) is an emerging technology, with the potential of becoming one of the future sources of sustainable energy. In MFCs waste is converted into electrons and protons via metabolic pathways of electroactive microbes, which release the charged particles during oxidation of organic compounds $[2,3]$. By introducing electrodes and a proton exchange membrane, the electrons can be captured and driven through an external circuit, generating green electricity.

This unique feature eliminates the technology's heavy reliance on environmental conditions, which remains a huge hindrance to success of other renewable energy technologies, and brings a circular economy element to the table, by making further use of waste. For this reason, the majority of MFC applications has targeted the wastewater treatment sector, where these devices are being used for the removal of organics, generating electricity on top [2].

Nonetheless, the technology is currently faced with numerous challenges related to its successful scale-up studies, with cost being the primary one [4-6]. The capital costs of scaled-up systems can range from USD $735 \mathrm{~m}^{-3}$ to USD $36,000 \mathrm{~m}^{-3}$. Electrode and membrane materials have been the main contributors to the high price of MFCs
[5,7]. In addition, operational costs such as pumping air and effluent, as well as mixing, have also been identified as detrimental to the energy usage and to the overall operational costs of the system [8]. For this reason, among the various types of MFC, soil microbial fuel cells (SMFCs) pose an interesting alternative.

SMFCs are characterized by an extremely simple design, with no need for fuel pumping and/or electrolyte replacement. Minimum maintenance is required since the organic matter is naturally provided in the soil by plants and/or decomposing matter, thus allowing long term operations [9]. The soil acts as a membrane, allowing membrane-less operation and the geometries and materials implemented are a lot cheaper compared to what used in conventional (i.e., liquid) MFCs [5].

Nonetheless, due to the relatively low power obtained from SMFCs, cost remains a hinderance in commercialising the technology. Our previous work, on a flat plate SMFC design [10], revealed that the cost of a single SMFC device is approximately $£ 2$. Titanium wire, used as the current collector (CC), was identified as responsible for up $50 \%$ of the total price. Furthermore, weaving the wire into the electrode resulted in the most time-consuming task in the fuel cell set-up. Therefore, with the aim of reducing the price and improving the practicality of SMFC installation, in this study we investigate the effect that a reduced amount of $\mathrm{CC}$ has on the overall performance of a flat SMFC.

\section{Materials and Methods}

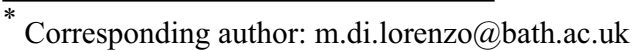




\subsection{Materials}

All reagents used were purchased of analytical grade from Alfa Aesar and Sigma-Aldrich, unless otherwise specified, and used without further purification. The soil was collected from the campus area of the University of Bath, and its characteristics are summarised in Table 1.

Table 1. Physiochemical properties of the soil

\begin{tabular}{ll}
\hline Parameter & \\
\hline $\mathbf{p H}$ & 6.5 \\
Conductivity & $195.2 \pm 23.3 \mu \mathrm{S} \mathrm{cm}^{-1}$ \\
Moisture content & $53 \%$ \\
Organic matter content & $17.4 \pm 1.5 \%$ \\
\hline
\end{tabular}

\subsection{SMFC design and operation}

Three flat plate, membrane-less air-cathode SMFC designs were constructed, as depicted in Figure 1A. Each design comprised of two graphite felt electrodes (anode and cathode), separated from each other by $4 \mathrm{~cm}$ with nylon screws. The designs differed from each other by the amount of $\mathrm{CC}$ used. The $\mathrm{CC}$, titanium wire $(0.25 \mathrm{~mm}$ diameter), was weaved through the electrodes, with High $\mathrm{CC}$, Medium CC and Low CC having respectively $32 \mathrm{~cm}$, $20 \mathrm{~cm}$ and $8 \mathrm{~cm}$ of wire, as depicted in Fig.1B-D. Each design had three replicates to evaluate reliability and reproducibility of the data obtained.

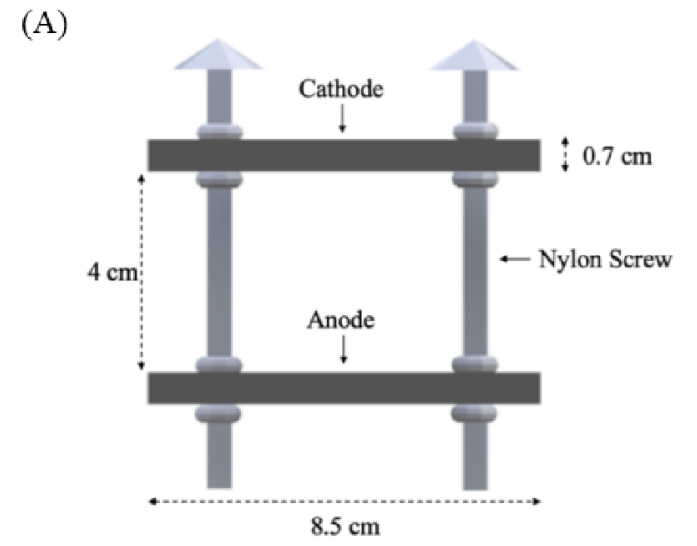

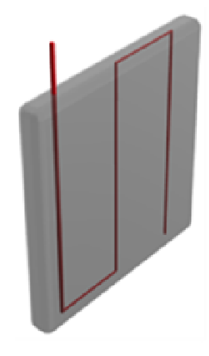

(B)

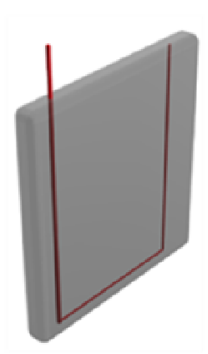

(C)

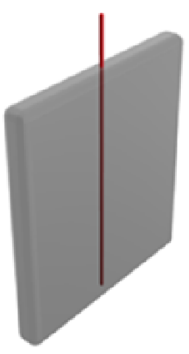

(D)
Fig.1: (A) Flat plate SMFC design; (B) High CC; (C) Medium CC; (D) Low CC

The SMFC reactors were fit into PVC containers ( 32 $\mathrm{x} 24 \mathrm{x} 10 \mathrm{~cm}$ ) filled up with soil that was kept moisturised with tap water. The anodes were buried inside the soil and the cathodes were exposed to air, as shown in Fig.2. The fuel cells were connected to an external resistance of 510 $\Omega$ and to a data acquisition system (DAQ6510, Keithley). Polarization tests were performed by connecting the SMFCs to a resistance box (Cropico RM6 Decade) and varying the applied resistance. Ohm's law (I=E/Rext) was used to calculate the current (I) corresponding to each externa resistance (Rext).

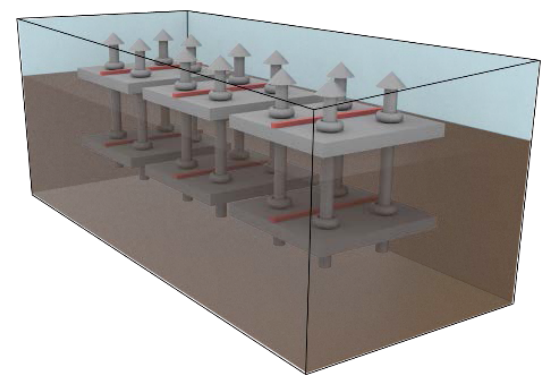

Fig.2: Example schematic of the experimental set-up showing 3 replicates of the Low CC SMFCs.

\section{Results}

\subsection{SMFC Performance}

The performance of the SMFCs was assessed and compared according to enrichment curves and individual electrode potentials. As shown in Fig.2A, the enrichment process clearly highlights the differences between the three configurations. The High CC SMFCs were the quickest to enrich, reaching a steady state of approximately $200 \mathrm{mV}$ after 20 days, followed by Medium CC (23 days) and Low CC (25 days). This difference is further reflected in the anode potentials, as shown in Fig.2B. The increased contact area between the current collector and the graphite felt, improves the electrode's electrical conductivity, widening the electrochemical signal reception from a larger part of electrode surface and lowering the charge transfer resistance. This strategy in turn facilitates the electron transfer and is likely the reason why a more rapid drop in the anode potential is observed [11]. Nonetheless, eventually all the configurations reached a comparable anode potential of $-400 \mathrm{mV}$ vs. $\mathrm{Ag} / \mathrm{AgCl}$ after 23 days, suggesting that the electrodes are inoculated with similar strains of electroactive species [12]. 
A)

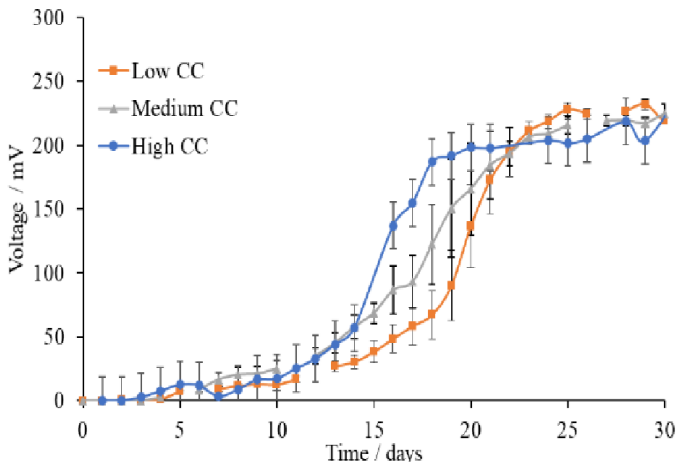

B)

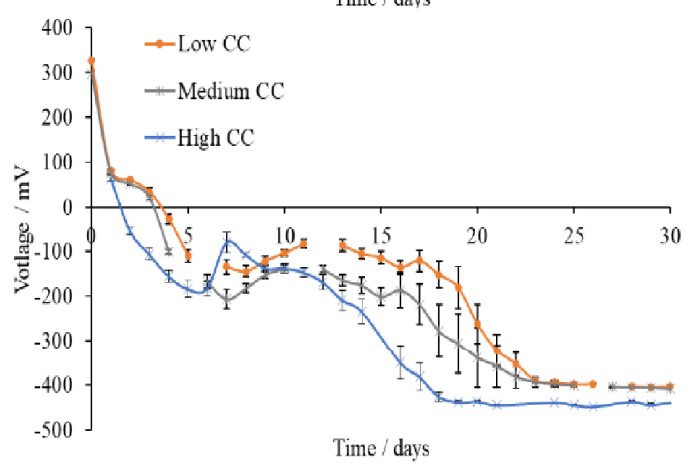

Fig.3: (A) Enrichment curves; (B) Anode electrode potentials vs. $\mathrm{Ag} / \mathrm{AgCl}$ reference electrode. Data is the average of 3 replicates, with error bars referring to the standard error.

Following the completion of the enrichment, a series of polarisation tests were conducted, to understand how much power can be generated by each SMFC. As the amount of titanium wire increased from $8 \mathrm{~cm}$ to $20 \mathrm{~cm}$ and then $32 \mathrm{~cm}$, the amount of power produced increased by $20 \%$ and $35 \%$ respectively (Fig.3). This result is attributed to the lower charge transfer resistance in High $\mathrm{CC}$ and Medium CC, due to a better contact of the CC with the electrode with respect to Low CC $[11,12]$. Capacitor characteristics of the electrodes could also play an important role. Paitier A. et al demonstrated that by increasing the amount of current collector, the capacitance of a microbial fuel cell increases leading to greater power densities during charge/discharge cycles [13].

\subsection{5}

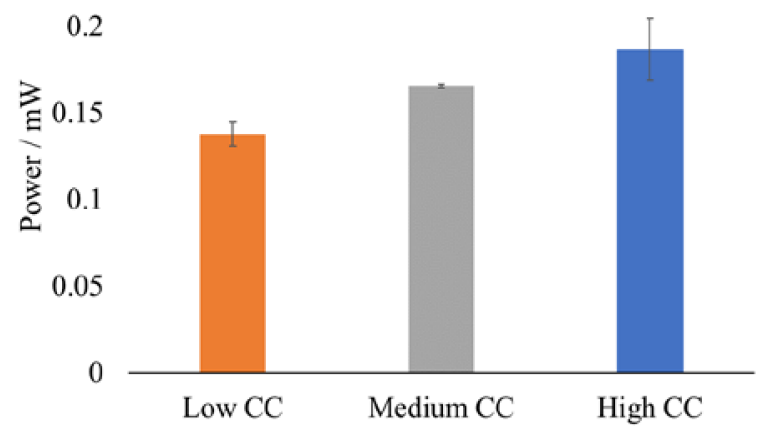

Fig.4: Bar chart of power generated by the different SMFCs. Error bars refer to three replicates.
Although increasing the amount of titanium wire has shown to enhance the power density of the flat-plate SMFC, such strategy remains questionable, due to the associated costs. At the current price point of titanium wire ( $£ 2.5$ per meter), increasing its amount by $150 \%$ and $300 \%$ would lead to a proportional increase in materials costs, yet only a $20 \%$ and $35 \%$ increase in performance, respectively. This relationship can be better described by representing it as cost per Watt of power, derived as shown by eq. 1-2.

$$
\begin{aligned}
& \text { Cost of CC used }(£)=\text { Amount of CC }(\mathrm{m}) * £ 2.5 / \mathrm{m} \\
& \text { Cost per Watt }=\frac{\text { Cost of CC used }(£)}{\text { Power }(\mathrm{mW})} * 1000 \frac{\mathrm{mW}}{\mathrm{W}}
\end{aligned}
$$

Considering that the power can be linearly scaled up by stacking the electrodes together [10], one can compare the cost per Watt with the amount of wire used in a single electrode (Fig.5). A scaled-up system comprising of Low CC SMFCs, would cost approximately $£ 1450$ per watt of power, whereas systems with Medium CC and High CC SMFCs would $£ 3,020$ and $£ 4,280$, respectively. This result reflects the disproportionality between the performance increase and the increase in material cost, proving Low $\mathrm{CC}$ to be most economically viable.

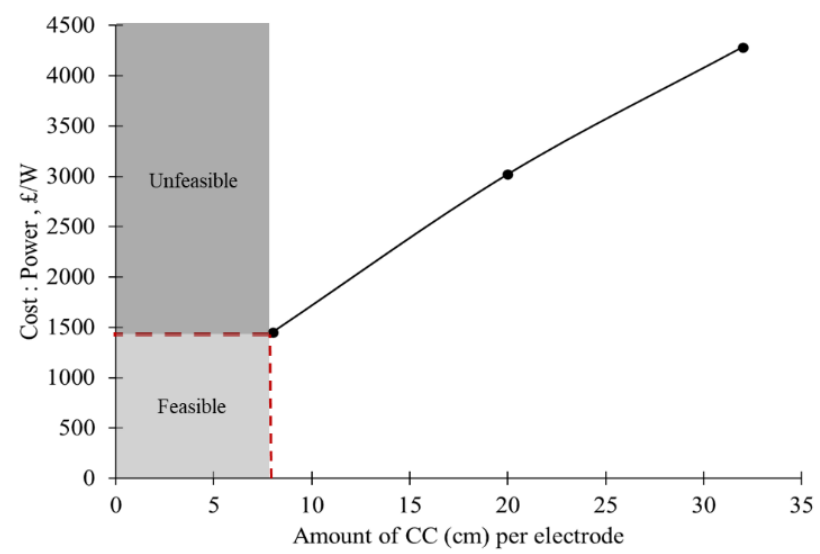

Fig.5: Relationship between the flat-plate SMFC cost per Watt of power $(£ / W)$ vs. the amount of current collector used.

To further optimise the cost of the SMFC device, a lower amount of titanium wire per electrode should be tested. In Figure 5, two regions have been identified, labelled as: Feasible and Unfeasible. The dotted red line represents the limit dictated by the Low CC configuration, of $£ 1,450$ per Watt, which indirectly sets the minimum power requirements to be generated by SMFCs with even less CC. For example, according to equations 1-2, a single $\mathrm{SMFC}$ with $4 \mathrm{~cm}$ of $\mathrm{CC}$ in each electrode should generate a minimum of $0.07 \mathrm{~mW}$, for the cost per Watt to be roughly $£ 1,450$. For an output power lower than this limit, the overall device cost would exceed the threshold, thus entering in a so-called unfeasible region. If the SMFC generates more than $0.07 \mathrm{~mW}$, the cost per Watt would accordingly drop, thus making the SMFC design more economically feasible.

\subsection{Cost Analysis}




\section{Conclusions}

Soil microbial fuel cells have great potential as energy harvesting technology for remote areas with minimum maintenance requirements. Cost-effective design solutions can markedly enhance scalability and implementation of this technology.

In this study, SMFC designs with different amounts of titanium, used as the current collector, were investigated. We showed that, while higher amounts of titanium wire would enhance the SMFC performance, the percentage increase in the power output is significantly smaller than the percentage increase in the material cost. From an economics standpoint, a large-scale system comprising of Low CC SMFCs would be significantly cheaper than Medium CC and High CC SMFC systems. Additional work is required to identify a point at which reducing the amount of $\mathrm{CC}$ begins to hinder the economics, in order to find the right balance between power output improvement and cost.

This project was funded by the UK Research England, under the Global Challenges Research Fund (GCFR), and is part of the project GREENER funded by the European Union's Horizon 2020 research and innovation programme under the grant agreement No 826312

\section{References}

[1] Lewis, C.W., Biomass (1981), 1, pp.,5-15.

[2] Logan, B.E. Microbial Fuel Cells; John Wiley \& Sons: Hoboken, NJ, USA, (2008).

[3] Dewan, A., Beyenal, H., Lewandowski, Z. Environ. Sci. Technol. (2008), 42, pp. 76437648.

[4] Liang, P., Duan, R., Jiang, Y., Zhang, X., Qiu, Y., Huang, X. Water Res. (2018), 141, pp. 18.

[5] Ge, Z., He, Z. Environ. Sci. Water Res. Technol. (2016), 2, pp. 274-281.

[6] Wang, A.-J., Wang, H.-C., Cheng, H.-Y., Liang, B., Liu, W.-Z., Han, J.-L., Zhang, B., Wang, S.-S., Environ. Sci. Ecotechnol. (2020).

[7] Rozendal, R.A., Hamelers, H.V., Rabaey, K., Keller, J., Buisman, C.J. Trends Biotechnol. (2008), 26, pp. 450-459. [CrossRef]

[8] Tommasi, T., Lombardelli, G. J. Power Sources (2017), 356, 438-447.
S. Maddalwar, K. Nayak, M. Kumar, L. Singh. Bioresour. Technol., 341, (2021)

[10] J. Dziegielowski, B. Metcalfe, P. Guzman, C.A. Martinez-Huitle, A. Gotrayeb, J. Wenk, M. Di Lorenzo, Appl Energy, 278 (2020)

[11] Malvankar, N. S., et al., Applied and Environmental Microbiology 78, 16, (2012)

[12] Commault, A. S., et al., Bioresour.Technol.139,226-234 (2013)

[13] Paitier, A., et al., Biotechnology. INSA Lyon, (2017) 